\title{
The association of genetic polymorphisms in interleukin- 1 receptors type 1 and type 2 with age-related hearing impairment in a Taiwanese population: a case control study
}

Ning-Chia Chang ${ }^{1,2,3}$, Hua-Ling Yang ${ }^{4}$, Chia-Yen Dai ${ }^{4,5,6}$, Wen-Yi Lin ${ }^{3}$, Hsun-Mo Wang ${ }^{2}$, Chen-Yu Chien ${ }^{1,7}$ and Kuen-Yao Ho ${ }^{1,7^{*}}$

\begin{abstract}
Background: Age-related hearing impairment (ARHI) is a major disability among the elder population. Chronic inflammation is an important factor in the development of ARHI. Interleukin-1 (IL-1) plays a key role in inflammation and may be associated with ARHI. The aim of this study is to analyze the associations of single nucleotide polymorphisms (SNPS) of IL-1 receptor genes with ARHI in an elderly population in Taiwan.

Method: Participants $\geq 65$ years of age were recruited for audiometric tests and genetic analyses. The bilateral puretone average (PTA) of high-tone hearing levels was calculated for ARHI evaluation. The associations of SNPs of the IL-1 receptor type 1 gene (IL1R1) (rs3917225 and rs2234650) and type 2 gene (IL1R2) (rs4141134 and rs2071008) with ARHI were analyzed in $182 \mathrm{ARHI}$-susceptible (case) and $176 \mathrm{ARHI}$-resistant (control) participants.

Results: The G allele of IL1R1 rs3917225 showed a decreased risk of ARHI after adjustments for sex, age, and noise exposure. The GG genotype of IL1R1 rs3917225 in all hereditary models and the TT genotype of IL1R2 rs2071008 in the recessive model also showed decreased risks of ARHI after adjustments.
\end{abstract}

Conclusion: These findings suggest that IL1R1 and IL1R2 polymorphisms may contribute to the decreased risk of $\mathrm{ARHI}$ in the elderly population.

Keywords: Age-related hearing impairment, Interleukin-1 receptor, Single nucleotide polymorphism

\section{Introduction}

Age-related hearing impairment (ARHI), or presbycusis, is one of the major disabilities among the elderly population. The prevalence of hearing impairment has been reported to be $34 \%$ in people 65 years of age or older, and it increases to $72 \%$ in people 85 years of age or older [1, 2]. In Taiwan, the prevalence of hearing impairment is

\footnotetext{
* Correspondence: kuyaho@kmu.edu.tw

'Department of Otorhinolaryngology, School of Medicine, College of Medicine, Kaohsiung Medical University, Kaohsiung, Taiwan

${ }^{7}$ Department of Otorhinolaryngology, Kaohsiung Medical University Hospital, No. 100, Tzyou 1st Road, Kaohsiung 807, Taiwan

Full list of author information is available at the end of the article
}

reportedly as high as $78 \%$ among the population 65 years of age or older [3]. Understanding the mechanisms of ARHI may help in the prevention and treatment of ARHI, hence decreasing its prevalence.

The detailed mechanisms of ARHI development remain unclear. Noise, toxic substances, hypoxia, and chronic inflammation may increase inner ear oxidative stress, produce reactive oxygen species (ROS), lead to the necrosis and apoptosis of inner ear cells, and result in presbycusis [4-6]. Experimental inner ear inflammation studies have shown the in vivo production of tumor necrosis factor- $\alpha$ (TNF- $\alpha$ ), interleukin-1 $\beta$ (IL-1 $\beta)$, and 
IL-6 in the cochlea, along with synergic leukocyte infiltration [7]. Interleukin-1 (IL-1) plays a key role in inflammation and autoimmune diseases by activating the expression of genes associated with the innate and adaptive immune response [8]. The IL-1 superfamily comprises the agonist IL- $1 \alpha$ and IL- $1 \beta$ and their antagonist IL-1Ra. Both IL-1 agonists can bind to IL-1 receptor type 1 and the "decoy" receptor type 2 [9]. Some authors found that the transplantation of the thymus into mice may alter the expression of IL-1 receptor type 2 on $\mathrm{CD} 4+\mathrm{T}$ cells and recover the hearing in mice with ARHI [10]. Accordingly, alternations in the expression of IL-1 receptor type 1 and type 2 may be associated with the development of ARHI.

The polymorphisms of the IL-1 receptor type 1 gene (IL1R1) and type 2 gene (IL1R2) have been reported to be associated with various diseases, such as knee osteoarthritis (OA), osteonecrosis of the femoral head, and IgA nephropathy [11-13].

In single nucleotide polymorphism (SNP) studies, the allele analysis and genotype analysis were performed. The allele analysis compared the frequency of the minor allele between the case and control groups. The genotype analysis investigated the association between the SNPs and target disease using multiple genetic models. The genetic models may include different inheritance patterns, such as codominant, dominant, and recessive. For example, in the knee OA study, the allele analysis demonstrated that the minor $G$ allele of SNP IL1R1 rs3917225 had a higher frequency in the knee OA patients than in the controls (41.3\% versus $33.7 \%$ ) and showed an increased risk of knee OA. And the minor G allele of IL1R1 rs3917225 was linked to an increased risk of knee OA based on the results of the dominant model [12].

However, no study on the association of IL1R1 and IL1R2 genetic polymorphisms with ARHI has been reported to date. We hypothesized that the genetic polymorphisms of interleukin-1 receptors may affect the individual's susceptibility to ARHI. The purpose of the present study was to investigate the association of IL1R1 and IL1R2 SNPs with ARHI in an elderly population in Taiwan.

\section{Material and methods Subjects}

The subjects of this study were recruited from the clients who received national annual health examinations performed by the health management center in a metropolitan hospital. The national annual health examinations were free of charge for subjects older than 65 years of age, with funding provided by the Health Promotion Administration, Ministry of Health and Welfare, Taiwan. We recruited volunteers who were 65 years of age or older and who agreed to participate in the study to receive additional pure-tone audiometric tests and fill out self-reported questionnaires about their medical history of ear diseases and history of noise exposures. Participants with histories of otologic diseases were excluded from this study later in the process of data analysis. Participants with dementia and those who could not sit independently to receive the audiometric tests were also excluded from this study.

\section{Audiometric assessments}

Pure-tone audiometry was performed in soundattenuating booths by trained medical technicians using standard procedures that met the requirements of the Council of Labor Affairs, Executive Yuan, Taiwan. The audiometric data were recorded at frequencies of 500 , 1000, 2000, 3000, 4000, 6000, and $8000 \mathrm{~Hz}$. The bilateral high-frequency $(4000,6000$, and $8000 \mathrm{~Hz})$ pure-tone average (PTA) was calculated. The participants were then divided into three groups according to the average high-frequency hearing levels. Participants in the worst quartile of hearing level were classified into the agerelated hearing impairment group (ARHI group), while those in the best quartile of hearing level were classified into the ARHI-resistant group. The others were classified into the intermediate group. The ARHI and ARHIresistant groups were chosen for case-control analysis.

\section{Genotyping}

IL1R1 and IL1R2 genetic polymorphisms rs3917225, rs2234650, rs4141134 and rs2071008 were selected as the target SNPs, which were referenced from an earlier study [14]. Blood samples were obtained from all the participants with written consent. Each specimen was collected in an ethylenediaminetetraacetic acid (EDTA) tube and centrifuged $(2000 \mathrm{~g}, 20 \mathrm{~min})$. The buffy coat was isolated, and DNA was extracted using a commercial DNA extraction kit (Gentra Corp., Minneapolis, Minn, USA). Genotypes for the selected polymorphisms were screened with the ABI TaqMan SNP genotyping assays (Applied Biosystems, Foster City, Calif., USA). The extracted DNA and genotyping assays were added to TaqMan universal PCR master mix (Roche, Branchburg, N.J., USA) according to the manufacturer's instructions. The genotyping procedures were then performed by using the ABI PRISM 7500 real-time PCR system (Applied Biosystems). The results were analyzed using ABI 7500 System sequence detection software version 1.2.3 (Applied Biosystems).

\section{Statistical analysis}

All data were input to a computer and analyzed using the $\mathrm{IBM}^{\circ} \mathrm{SPSS}^{\circ}$ statistics software package version 20.0 (International Business Machines Corp., Armonk, N.Y., 
USA). Continuous data were analyzed using the independent-sample Student's t-tests. Categorical data were computed using the two-sided $\chi^{2}$ test. Genetic analyses were performed using PLINK software [15]. The calculation of odds ratios (ORs) and 95\% confidence intervals $(95 \% \mathrm{CI})$ and the adjustments for potentially confounding factors were performed with logistic regressions. The level of statistical significance was set at $p<0.05$.

\section{Results}

A total of 713 volunteers, including 400 men (56.1\%) and 313 women $(43.9 \%)$, were included in the present study. The mean age of the participants was $72.04 \pm$ 5.96 years (range 65-97 years). The average high-tone hearing level was $62.36 \pm 19.92 \mathrm{~dB}$ (range 16.67$111.67 \mathrm{dBHL}$ ). Among the participants, 232 (32.5\%) people claimed to have been exposed to occupational noises over $85 \mathrm{dBSPL}$ before retirement. In the participants who were exposed to occupational noises, 178 were men $(44.5 \%$ of the male participants; $76.7 \%$ of total noise-exposed participants), and 54 were women (17.3\% of the female participants; $23.3 \%$ of total noise-exposed participants). Other demographic data are presented in Table 1 .

\section{Case-control study}

There were $182(25.5 \%)$ participants classified into the ARHI-susceptible group as cases (mean age $=75.34 \pm$ 6.48 years; average PTA $=88.19 \pm 10.39 \mathrm{dBHL}$; male $/ \mathrm{fe}-$ male $=142 \quad(78.0 \%) / 40 \quad(22.0 \%))$, and $176 \quad(24.7 \%)$ participants with the best hearing, classified into the ARHI-resistant group as the controls (mean age = $68.92 \pm 4.21$ years; average $\mathrm{PTA}=37.44 \pm 8.01 \mathrm{dBHL}$; male $/$ female $=61 \quad(34.7 \%) / 115 \quad(65.3 \%))$. The participants in the control group were significantly younger than those in the case group ( $\mathrm{t}$ test, $p<0.001$ ). The sex structure was quite different between the case and control groups, in which women had better hearing than men (chi-square; $p<0.001$ ). The distribution of noise exposure history $(\mathrm{Y} / \mathrm{N})$ was also different between these two groups. There were more participants with noise exposure history in ARHI group (38.5\%) than those in the control group (22.2\%) (chisquare; $p=0.001$ ).

\section{Genetic analyses Allele analysis}

Our analyses showed no significant association of the alleles of SNPs rs2234650, rs4141134 or rs2071008 with ARHI susceptibility in the present study, regardless of the adjustments for sex, age and noise exposure. Although there was no significant association of rs3917225 with ARHI susceptibility in the crude
Table 1 Demographics of the participants

\begin{tabular}{llll}
\hline & Male & Female & Total \\
\hline Number & $400(56.1 \%)$ & $313(43.9 \%)$ & $713(100 \%)$ \\
Age (Years) & $72.42 \pm 6.14$ & $71.56 \pm 5.71$ & $72.04 \pm 5.96$ \\
$\begin{array}{l}\text { Bilateral PTA average } \\
\text { (dBHL) }\end{array}$ & $67.96 \pm 18.87$ & $55.27 \pm 18.93$ & $62.36 \pm 19.92$ \\
$\begin{array}{l}\text { Occupational noise } \\
\text { exposure }\end{array}$ & $178(44.5 \%)$ & $54(17.3 \%)$ & $232(32.5 \%)$ \\
$\begin{array}{l}\text { ARHI grouping } \\
\quad \text { ARHI susceptible }\end{array}$ & $142(35.5 \%)$ & $40(12.8 \%)$ & $182(25.5 \%)$ \\
$\quad$ Intermediate & $197(49.2 \%)$ & $158(50.5 \%)$ & $355(49.8 \%)$ \\
$\quad$ ARHI resistant & $61(15.2 \%)$ & $115(36.7 \%)$ & $176(24.7 \%)$ \\
Genotype & & & \\
$\quad$ IL1R1 & & &
\end{tabular}

rs3917225

$\begin{array}{llll}\text { AA } & 130(32.5 \%) & 112(35.8 \%) & 242(33.9 \%) \\ \text { AG } & 188(47.0 \%) & 137(43.8 \%) & 325(45.6 \%) \\ \text { GG } & 60(15.0 \%) & 46(14.7 \%) & 106(14.9 \%) \\ \text { Undetermined } & 22(5.5 \%) & 18(5.8 \%) & 40(5.6 \%) \\ \text { rs2234650 } & & & \\ \text { CC } & 225(56.2 \%) & 146(46.6 \%) & 371(52.0 \%) \\ \text { CT } & 141(35.2 \%) & 136(43.5 \%) & 277(38.8 \%) \\ \text { TT } & 25(6.2 \%) & 20(6.4 \%) & 45(6.3 \%) \\ \text { Undetermined } & 9(2.8 \%) & 11(3.5 \%) & 20(2.8 \%)\end{array}$

IL1R2

rs4141134

Tा

$\mathrm{TC}$

$356(89.0 \%)$

$277(88.5 \%)$

$633(88.8 \%)$

CC

$32(8.0 \%)$

$21(6.7 \%)$

$53(7.4 \%)$

$0(0)$

$0(0)$

$0(0)$

Undetermined

$12(3.0 \%)$

15 (4.8\%)

27 (3.8\%)

rs2071008

$\begin{array}{llll}\text { GG } & 223(55.8 \%) & 188(60.1 \%) & 411(57.6 \%) \\ \text { GT } & 138(34.5 \%) & 99(31.6 \%) & 237(33.2 \%) \\ \text { TT } & 16(4.0 \%) & 13(4.2 \%) & 29(4.1 \%) \\ \text { Undetermined } & 23(5.8 \%) & 13(4.2 \%) & 36(5.0 \%)\end{array}$

Allele

IL1R1

rs3917225

A

448 (59.3\%)

361 (61.2\%)

809 (60.1\%)

G

308 (40.7\%)

229 (38.8\%)

537 (39.9\%)

rs2234650

C

591 (75.6\%)

428 (70.9\%)

1019 (73.5\%)

$\mathrm{T}$

191 (24.4\%)

176 (29.1\%)

367 (26.5\%)

IL1R2

rs4141134
T
C


Table 1 Demographics of the participants (Continued)

\begin{tabular}{clll}
\hline & Male & Female & Total \\
\hline rs2071008 & & & \\
$G$ & $584(77.5 \%)$ & $475(79.2 \%)$ & $1059(78.2 \%)$ \\
$T$ & $170(22.5 \%)$ & $125(20.8 \%)$ & $295(21.8 \%)$ \\
\hline
\end{tabular}

analysis, it revealed a significant association between rs3917225 and ARHI susceptibility after the adjustments. Participants with the G allele of rs3917225 had a decreased risk of ARHI (adjusted OR $=0.587$; 95\% CI $=0.383-0.901 ; p=0.015$ ). The results of allele analyses are presented in Table 2 . The genetic distribution in the present study followed Hardy-Weinberg equilibrium.

\section{Genotype analysis}

Multiple genetic models, including codominant, dominant, and recessive inheritance patterns, were used in the genotype analysis. The analyses of genotypes showed no significant difference in the target SNPs between groups in the crude analysis. However, after adjusting for sex, age and noise exposure, rs3917225 and rs2071008 showed significant associations with ARHI. The participants with the homozygous GG genotype at rs3917225 had a decreased risk of ARHI, regardless of the inheritance model, after the adjustments. At rs2071008, the TT genotype was associated with a lower risk of ARHI in the recessive inherence model after the adjustments (adjusted OR $=0.187 ; 95 \% \mathrm{CI}=0.037-0.944 ; p=0.042$ ). There was no significant association under any inheritance model of SNP rs2234650 or rs4141134 with ARHI susceptibility, regardless of the adjustments for sex, age and noise exposure. The results of the genotype analyses are presented in Table 3.

\section{Discussion}

ARHI is a multifactorial condition representing the end result of multiple intrinsic (e.g., genetic predisposition) and extrinsic factors (e.g., noise exposure) acting on the inner ear and leading to the accumulation of damages in the pathway of auditory signal transduction [6]. Chronic inflammation, hypoxia, noise, and toxic substances may increase oxidative stress in the inner ear, cause the production of reactive oxygen species, lead to necrosis and apoptosis of inner ear cells, and result in ARHI [4-6]. In the present study, we confirmed the hypothesis that the genetic polymorphisms of IL-1 receptors are associated with susceptibility to ARHI. These results suggest that the development of ARHI is related to the inflammatory pathway and is underlain by individual genetic differences.

IL-1 is a central mediator of innate immunity and inflammation. As a multifunctional proinflammatory cytokine, IL-1 plays a key role in inflammation and autoimmune diseases by activating the expression of genes associated with the innate and adaptive immune response $[8,16]$. The biological activity of the cytokine IL-1 is medicated by its receptors [17]. IL-1 receptor type 1 affects NF- $\mathrm{kB}$ signaling by combining with IL-1 on the cell surface and upregulates inflammation [18]. The IL1R1 and IL1R2 genes encode cytokine receptors for IL-1. A number of studies have been performed to

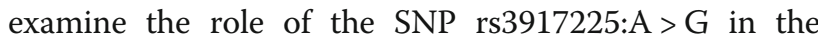
IL1R1 gene. Nakki et al. [19] showed that the G allele is associated with a decreased risk of severe hand osteoarthritis. $\mathrm{Na}$ et al. [12] found that the G allele increases the risk of knee osteoarthritis. In our study, the homozygous variant GG genotype of rs3917225 was associated with a decreased risk of ARHI, but the AG genotype showed an insignificant association with ARHI. The possible explanation might be that the GG genotype of rs3917225 decreased the risk of ARHI in the recessive pattern, and the effect of GG genotype overcame the effect of the AG genotype, resulted in the significance in the dominant model. It is not clear how this SNP affects the development of ARHI. Vasilyev et al. [14] analyzed the relationship between IL1R1 genetic polymorphisms

Table 2 Relationships between alleles of IL-1 receptor genetic polymorphisms and age-related hearing impairment

\begin{tabular}{|c|c|c|c|c|c|c|c|c|}
\hline \multirow[t]{2}{*}{ Gene } & \multirow[t]{2}{*}{ SNPS } & \multirow[t]{2}{*}{ Allele } & \multirow[t]{2}{*}{ ARHI } & \multirow[t]{2}{*}{ Control } & \multicolumn{2}{|l|}{ Crude } & \multicolumn{2}{|l|}{ Adjusted $^{a}$} \\
\hline & & & & & OR $(95 \% \mathrm{Cl})$ & $P$ & $\mathrm{OR}(95 \% \mathrm{Cl})$ & $p$ \\
\hline \multirow[t]{4}{*}{ IL1R1 } & rs3917225 & A & $216(63.2 \%)$ & $186(57.1 \%)$ & 1.000 & & 1.000 & \\
\hline & & G & $126(36.8 \%)$ & $140((42.9 \%)$ & $0.775(0.568-1.057)$ & 0.107 & $0.587(0.383-0.901)$ & $0.015^{*}$ \\
\hline & rs2234650 & C & $270(75.4 \%)$ & $225(71.7 \%)$ & 1.000 & & 1.000 & \\
\hline & & $\mathrm{T}$ & $88(24.6 \%)$ & $89(28.3 \%)$ & $0.934(0.664-1.313)$ & 0.694 & $1.028(0.654-1.616)$ & 0.905 \\
\hline \multirow[t]{4}{*}{ IL1R2 } & rs4141134 & $\mathrm{T}$ & 343 (96.3\%) & $333(96.2 \%)$ & 1.000 & & 1.000 & \\
\hline & & C & $13(3.7 \%)$ & $13(3.8 \%)$ & $0.971(0.444-2.125)$ & 0.941 & $0.595(0.209-1.699)$ & 0.332 \\
\hline & rs2071008 & G & $276(78.4 \%)$ & $261(79.6 \%)$ & 1.000 & & 1.000 & \\
\hline & & $\mathrm{T}$ & $76(21.6 \%)$ & $67(20.4 \%)$ & $1.073(0.741-1.552)$ & 0.710 & $1.062(0.641-1.760)$ & 0.816 \\
\hline
\end{tabular}

a: Logistic regression; adjusted for age, sex and noise exposure

*: $p<0.05$ 
Table 3 Relationships between genotypes of IL-1 receptor genetic polymorphisms and age-related hearing impairment

\begin{tabular}{|c|c|c|c|c|c|c|c|c|c|}
\hline \multirow[t]{2}{*}{ Gene } & \multirow[t]{2}{*}{ SNP } & \multirow[t]{2}{*}{ Model } & \multirow[t]{2}{*}{ Genotype } & \multirow[t]{2}{*}{ ARHI } & \multirow[t]{2}{*}{ Control } & \multicolumn{2}{|l|}{ Crude } & \multicolumn{2}{|l|}{ Adjusted $^{a}$} \\
\hline & & & & & & $\overline{\mathrm{OR}}(95 \% \mathrm{Cl})$ & $p$ & OR $(95 \% \mathrm{Cl})$ & $p$ \\
\hline \multirow[t]{14}{*}{ IL1R1 } & rs3917225 & Codominant & AA & 66 (38.6\%) & $53(32.5 \%)$ & 1.000 & & 1.000 & \\
\hline & & & $A G$ & $84(49.1 \%)$ & $80(49.1 \%)$ & $0.843(0.525-1.354)$ & 0.480 & $0.637(0.329-1.232)$ & 0.180 \\
\hline & & & GG & $21(12.3 \%)$ & 30 (18.4\%) & $0.562(0.289-1.093)$ & 0.089 & $0.374(0.146-0.956)$ & $0.040^{*}$ \\
\hline & & Dominant & AA & $66(38.6 \%)$ & $53(32.5 \%)$ & 1.000 & & 1.000 & \\
\hline & & & $A G+G G$ & $105(61.4 \%)$ & $110(67.5 \%)$ & $0.767(0.489-1.202)$ & 0.246 & $0.546(0.301-0.991)$ & $0.047^{*}$ \\
\hline & & Recessive & $A A+A G$ & $150(87.7 \%)$ & $133(81.6 \%)$ & 1.000 & & 1.000 & \\
\hline & & & GG & $21(12.3 \%)$ & 30 (18.4\%) & $0.621(0.339-1.136)$ & 0.122 & $0.420(0.182-1.966)$ & $0.041^{*}$ \\
\hline & rs 2234650 & Codominant & CC & $100(55.9 \%)$ & 91 (52.9\%) & 1.000 & & 1.000 & \\
\hline & & & CT & 70 (39.1\%) & 73 (42.4\%) & $0.882(0.565-1.378)$ & 0.583 & $0.982(0.542-1.780)$ & 0.952 \\
\hline & & & $T$ & $9(5.0 \%)$ & $8(4.7 \%)$ & $0.511(0.149-1.758)$ & 0.287 & $0.526(0.127-2.171)$ & 0.374 \\
\hline & & Dominant & CC & $100(55.9 \%)$ & 91 (52.9\%) & 1.000 & & 1.000 & \\
\hline & & & $C T+\pi$ & 79 (44.1\%) & $81(47.1 \%)$ & $0.888(0.583-1.351)$ & 0.578 & $1.012(0.582-1.759)$ & 0.966 \\
\hline & & Recessive & $C C+C T$ & $170(95.0 \%)$ & $164(95.3 \%)$ & 1.000 & & 1.000 & \\
\hline & & & $\pi$ & $9(5.0 \%)$ & $8(4.7 \%)$ & $1.085(0.409-2.881)$ & 0.869 & $1.098(0.348-3.463)$ & 0.873 \\
\hline \multirow[t]{14}{*}{ IL1R2 } & rs4141134 & Codominant & $\pi$ & $165(92.7 \%)$ & $160(92.5 \%)$ & 1.000 & & 1.000 & \\
\hline & & & $\mathrm{TC}$ & $13(7.3 \%)$ & $13(7.5 \%)$ & $0.940(0.408-2.165)$ & 0.885 & $0.500(0.163-1.535)$ & 0.226 \\
\hline & & & CC & $0(0)$ & $0(0)$ & NA & NA & NA & NA \\
\hline & & Dominant & $\pi$ & $165(92.7 \%)$ & 160 (92.5\%) & 1.000 & & 1.000 & \\
\hline & & & $\mathrm{TC}+\mathrm{CC}$ & $13(7.3 \%)$ & $13(7.5 \%)$ & $0.940(0.408-2.165)$ & 0.885 & $0.500(0.163-1.535)$ & 0.226 \\
\hline & & Recessive & $T+T C$ & $178(100 \%)$ & $173(100 \%)$ & 1.000 & & 1.000 & \\
\hline & & & CC & $0(0)$ & $0(0)$ & NA & NA & NA & NA \\
\hline & rs2071008 & Codominant & GG & $104(59.1 \%)$ & $104(63.4 \%)$ & 1.000 & & 1.000 & \\
\hline & & & GT & $68(38.6 \%)$ & $53(32.3 \%)$ & $1.224(0.768-1.950))$ & 0.395 & $1.416(0.769-2.609)$ & 0.264 \\
\hline & & & $\pi$ & $4(2.3 \%)$ & $7(4.3 \%)$ & $0.479(0.120-1.907)$ & 0.297 & $0.190(0.032-1.110)$ & 0.065 \\
\hline & & Dominant & GG & $104(59.1 \%)$ & $104(63.4 \%)$ & 1.000 & & 1.000 & \\
\hline & & & $\mathrm{GT}+\mathrm{TT}$ & 72 (40.9\%) & $60(36.6 \%)$ & $1.200(0.775-1.858)$ & 0.414 & $1.294(0.736-2.227)$ & 0.370 \\
\hline & & Recessive & $\mathrm{GG}+\mathrm{GT}$ & $172(97.7 \%)$ & 157 (95.7\%) & 1.000 & & 1.000 & \\
\hline & & & TT & $4(2.3 \%)$ & 7 (4.3\%) & $0.522(0.150-1.816)$ & 0.306 & $0.187(0.037-0.944)$ & $0.042^{*}$ \\
\hline
\end{tabular}

a: Logistic regression; adjusted for age, sex and noise exposure

*: $p<0.05$

and the expression level of membrane-bound receptors and found that rs3917225 did not show any association with the expression level of IL1R1. Dinarello et al. [20] found that IL1R1 was not abundantly expressed on the cell surface. These findings suggest that rs3917225 does not change the quantity of IL1R1, but it may alter some quality of the receptor instead. We postulate that this genetic variant might affect the affinity of IL-1 to IL-1 receptor 1, hence blocking the upregulation of inflammation.

Interleukin-1 receptor 2 is a molecular decoy that traps IL-1 $\beta$ and does not initiate subsequent signaling events, thereby suppressing the inflammatory response $[11,13]$. Many studies have reported the associations of IL1R2 with certain diseases. Xie et al. [13] reported that the GA genotype of rs4851527:A > G exhibited increased IgA nephropathy (IgAN) risk, whereas the GA genotype of rs3218977:G > A showed decreased IgAN risk. Another study reported that the IL1R2 SNP rs11674595: $\mathrm{T}>\mathrm{C}$ showed an increased risk of osteonecrosis of the femoral head $(\mathrm{ONFH})$ [11]. It was reported that the minor allele $\mathrm{T}$ of rs2071008: $\mathrm{T}>\mathrm{G}$ decreased the risk of coronary heart disease in younger adults $(\leq 55$ years of age) in the study by Chen et al. [21]. In our study, we found that rs2071008 decreased the risk of ARHI in a recessive hereditary pattern. The finding that the TT genotype of rs2071008 decreased ARHI risk in the present study was similar to the result from the study mentioned above. In the study of Vasilyev et al., homozygotes for the major allele of rs2071008 showed a lower level of 
membrane-bound IL1R2 on $\mathrm{CD}^{+} 4^{+}$monocytes in lipopolysaccharide-stimulated PBMC cultures [14]. From previous findings, we postulate that the individuals with the TT genotype of rs2071008 may have a higher level of membrane-bound IL1R2 for IL-1 binding, hence enhancing the effect of inflammation suppression.

The association of SNPs with the disease may be organ specific. In an Iranian study, the authors reported that some haplotypes of SNPs IL-1A rs1800587, IL-1B rs1143634 and IL1R1 rs2234650 were associated with systemic sclerosis [22]. Another study found that the haplotype of three IL1R2 SNPs rs4141134, rs11674595, and rs7570441 was associated with an increased risk on depressive symptom trajectories in oncology patients and family caregivers [23]. These reports indicated that IL1R1 rs2234650 might be associated with muscular diseases while IL1R2 rs4141134 might be relating to psychologic diseases. However, no association of IL1R1 rs2234650 and IL1R2 rs4141134 with ARHI was found in the present study.

Several limitations existed in the present study. First, the sample size of our study was relatively small (182 cases and 176 controls). Second, although the history of noise exposure was adjusted during static analyses, the detailed time and dose of noise exposed could not be ascertained. More male participants had occupational noise exposure and hearing loss than females. The interaction of sex and noise exposure on ARHI might be another confounding factor that should be taken into consideration. The effects of age, sex, and noise exposure might be too enormous that masks the effect of SNPs on ARHI. Therefore, we adjusted for age, sex, and noise exposure history simultaneously in the regression analysis. We believed that the results from the adjusted analysis may be closer to the true associations of SNPs with ARHI. Another limitation was that other potential risk factors of hearing loss (e.g., the habit of smoking) could not be integrated and analyzed in the present study.

In summary, we found that SNPs rs3917225 of IL1R1 and rs2071008 of IL1R2 were associated with a decreased risk of ARHI. The effect of ARHI protection might be via inflammation suppression by either decreasing the affinity of IL-1 to the IL1R1 receptor or increasing the presence of membrane-bound IL1R2 for IL-1 to bind. In a phase I/II clinical trial, the authors reported that anakinra (an IL-1 receptor antagonist) therapy in corticosteroid-resistant autoimmune inner ear disease patients was effective in a small cohort of patients and that plasma IL- $1 \beta$ level was associated with both clinical hearing response and disease relapse [24]. Anti-inflammatory agents such as IL-1 receptor antagonists may have potential in the prevention and/or treatment of ARHI and are worth further study.

\section{Conclusion}

In the present study, we found that the genetic polymorphisms of IL1R1 and IL1R2 were associated with ARHI in an elderly population in Taiwan. The minor G allele and GG genotype of SNP IL1R1 rs3917225 and the homozygous variant TT genotype of IL1R2 rs2071008 in recessive inheritance pattern demonstrated decreased risks of ARHI. In contrast, SNPs rs2234650 of IL1R1 and rs4141134 of IL1R2 showed no association with ARHI.

\section{Acknowledgements \\ We thank the Teaching and Research Center of Kaohsiung Municipal Siaogang Hospital for supporting this study with the grant number kmhk- 107-006 and the Department of Medical Research of Kaohsiung Medical Uni- versity Hospital for the grant KMUH106-6 M40.}

\section{Authors' contributions}

Draft of the manuscript (Chang NC), specimen and data analysis (Yang $\mathrm{HL}$, Wang HM), data collection (Dai CY, Lin WY), review of the manuscript (Chien $\mathrm{CY}$ ), supervisor of the project and final approval of the manuscript (Ho KY). The author(s) read and approved the final manuscript.

\section{Funding}

Not applicable.

Availability of data and materials Not applicable.

\section{Ethics approval and consent to participate}

All participants provided written informed consent. No private personal information was identifiable in the data. This study was approved by the institutional review board of our institute (IRB approval No.: KMUHIRB-G(I)20150011).

\section{Consent for publication}

Not applicable.

\section{Competing interests}

The authors declare that they have no competing interests.

\section{Author details}

'Department of Otorhinolaryngology, School of Medicine, College of Medicine, Kaohsiung Medical University, Kaohsiung, Taiwan. ${ }^{2}$ Department of Otorhinolaryngology, Kaohsiung Municipal Siaogang Hospital, Kaohsiung,

Taiwan. ${ }^{3}$ Health Management Center, Kaohsiung Municipal Siaogang Hospital, Kaohsiung, Taiwan. ${ }^{4}$ Division of Hepatobiliary and Pancreatic Medicine, Department of Internal Medicine, Kaohsiung Medical University Hospital, Kaohsiung, Taiwan. ${ }^{5}$ Department of Internal Medicine, School of Medicine, College of Medicine, Kaohsiung Medical University, Kaohsiung, Taiwan. ${ }^{6}$ Health Management Center, Kaohsiung Medical University Hospital, Kaohsiung, Taiwan. ${ }^{7}$ Department of Otorhinolaryngology, Kaohsiung Medical University Hospital, No. 100, Tzyou 1st Road, Kaohsiung 807, Taiwan.

Received: 20 October 2019 Accepted: 18 March 2020

Published online: 06 April 2020

\section{References}

1. Gates GA, Cooper JC Jr, Kannel WB, Miller NJ. Hearing in the elderly: the Framingham cohort, 1983-1985. Part I. basic audiometric test results. Ear Hear. 1990;11(4):247-56.

2. Pacala JT, Yueh B. Hearing deficits in the older patient: "I didn't notice anything". JAMA. 2012;307(11):1185-94.

3. Chang N-C, Dai C-Y, Lin W-Y, Chien C-Y, Hsieh M-H, Ho K-Y. Perception of hearing impairment and the willingness to use hearing aids in an elderly population in southern Taiwan: a community-based study. Int J Audiol. 2016;55(9):491-8. 
4. Bared A, Ouyang X, Angeli S, Du LL, Hoang K, Yan D, et al. Antioxidant enzymes, presbycusis, and ethnic variability. Otolaryngol Head Neck Surg. 2010;143(2):263-8.

5. Henderson D, Bielefeld EC, Harris KC, Hu BH. The role of oxidative stress in noise-induced hearing loss. Ear Hear. 2006;27(1):1-19.

6. Yamasoba T, Lin FR, Someya S, Kashio A, Sakamoto T, Kondo K. Current concepts in age-related hearing loss: epidemiology and mechanistic pathways. Hear Res. 2013;303:30-8.

7. Hashimoto S, Billings P, Harris JP, Firestein GS, Keithley EM. Innate immunity contributes to cochlear adaptive immune responses. Audiol Neurootol. 2005:10(1):35-43.

8. Dinarello CA. The IL-1 family and inflammatory diseases. Clin Exp Rheumatol. 2002;20(5 Suppl 27):S1-13.

9. Dinarello CA. Interleukin-1, interleukin-1 receptors and interleukin-1 receptor antagonist. Int Rev Immunol. 1998;16(5-6):457-99.

10. Iwai $\mathrm{H}$, Inaba M. Fetal thymus graft enables recovery from age-related hearing loss and expansion of CD4-Positive T cells expressing IL-1 receptor type 2 and regulatory T Cells. Immun Ageing. 2015;12(1):26.

11. An F, Wang J, Gao H, Liu C, Tian Y, Jin T, et al. Impact of IL1R1 and IL1R2 gene polymorphisms on risk of osteonecrosis of the femoral head from a case-control study. Mol Genet Genomic Med. 2019;7(3):e00557.

12. Na Y, Bai R, Zhao Z, Wei Y, Li D, Wang Y, et al. IL1R1 gene polymorphisms are associated with knee osteoarthritis risk in the Chinese Han population. Oncotarget. 2017:8(3):4228-33.

13. Xie M, Zhang D, Zhang Y, Yang $X$, Su Y, Wang Y, et al. Association of genetic polymorphisms in IL-1R1 and IL-1R2 genes with IgA nephropathy in the Han Chinese population. Oncotarget. 2017:8(31):50673-9.

14. Vasilyev FF, Silkov AN, Sennikov SV. Relationship between interleukin-1 type 1 and 2 receptor gene polymorphisms and the expression level of membrane-bound receptors. Cell Mol Immunol. 2014;12(2):222-30.

15. Purcell S, Neale B, Todd-Brown K, Thomas L, Ferreira MA, Bender D, et al. PLINK: a tool set for whole-genome association and population-based linkage analyses. Am J Hum Genet. 2007;81(3):559-75.

16. Garlanda C, Dinarello CA, Mantovani A. The interleukin-1 family: back to the future. Immunity. 2013;39(6):1003-18.

17. Lukens JR, Gross JM, Kanneganti TD. IL-1 family cytokines trigger sterile inflammatory disease. Front Immunol. 2012;3:315.

18. Rhodes DM, Smith SA, Holcombe M, Qwarnstrom EE. Computational Modelling of NF-kappaB activation by IL-1RI and its co-receptor TILRR, predicts a role for cytoskeletal sequestration of IkappaBalpha in inflammatory Signalling. PLoS One. 2015;10(6):e0129888.

19. Nakki A, Kouhia ST, Saarela J, Harilainen A, Tallroth K, Videman T, et al. Allelic variants of ILIR1 gene associate with severe hand osteoarthritis. BMC Med Genet. 2010;11:50.

20. Dinarello CA. Biologic basis for interleukin-1 in disease. Blood. 1996; 87(6):2095-147.

21. Chen $X$, Chen $X, X u Y$, , Yang W, Wu N, Ye H, et al. Association of six CpGSNPs in the inflammation-related genes with coronary heart disease. Hum Genomics. 2016;10(Suppl 2):21.

22. Abtahi S, Farazmand A, Mahmoudi M, Ashraf-Ganjouei A, Javinani A, Nazari B, et al. IL-1A rs1800587, IL-1B rs1 143634 and IL-1R1 rs2234650 polymorphisms in Iranian patients with systemic sclerosis. Int J Immunogenet. 2015;42(6):423-7.

23. Dunn LB, Aouizerat BE, Langford DJ, Cooper BA, Dhruva A, Cataldo JK et al. Cytokine gene variation is associated with depressive symptom trajectories in oncology patients and family caregivers. Eur J Oncol Nurs. 2013;17(3):346-53.

24. Vambutas A, Lesser M, Mullooly V, Pathak S, Zahtz G, Rosen L, et al. Early efficacy trial of anakinra in corticosteroid-resistant autoimmune inner ear disease. J Clin Invest. 2014;124(9):4115-22.

\section{Publisher's Note}

Springer Nature remains neutral with regard to jurisdictional claims in published maps and institutional affiliations.

Ready to submit your research? Choose BMC and benefit from:

- fast, convenient online submission

- thorough peer review by experienced researchers in your field

- rapid publication on acceptance

- support for research data, including large and complex data types

- gold Open Access which fosters wider collaboration and increased citations

- maximum visibility for your research: over $100 \mathrm{M}$ website views per year

At BMC, research is always in progress.

Learn more biomedcentral.com/submissions 